\title{
HABILIDADES MATERNAS PARA PREVENÇÃO E MANEJO DA DIARREIA INFANTIL
}

\author{
MATERNAL ABILITIES IN THE PREVENTION AND HANDLING \\ OF INFANTILE DIARRHEA
}

\section{HABILIDADES MATERNAS PARA PREVENCIÓN Y MANEJO DE LA DIARREA INFANTIL}

\author{
Emanuella Silva Joventino* \\ Lydia Vieira Freitas ** \\ Neiva Francenely Cunha Vieira ${ }^{* * *}$ \\ PRISCILA DE SOUZA AQUino ${ }^{* * * *}$ \\ Ana Karina Bezerra Pinheiro ${ }^{* * * * *}$ \\ Lorena Barbosa Ximenes ${ }^{* * * * *}$
}

\begin{abstract}
RESUMO
Objetivo:Identificar as habilidades maternas na prevenção e manejo da diarreia infantil. Materiale e método: Estudo transversal, quantitativo, realizado com 448 mães de crianças menores de 5 anos residentes em Fortaleza-CE. A coleta de dados ocorreu por meio de entrevista, utilizando-se um formulário abordando características sociodemográficas e aspectos relacionados ao cuidado materno para prevenção e manejo da diarreia em seus filhos. Resultados: As mães tinham em média 28,34 anos e 9,1 anos de estudo. A mediana da renda per capta foi $\mathrm{R} \$ 150$. A maioria possuía companheiro, trabalhava em casa e morava há mais de cinco anos na mesma residência. Verificou-se correlação estatisticamente significante entre a realização de receita caseira e idade materna ( $\mathrm{p}=0,027)$; entre oferta de solução de reidratação oral para a criança e anos de estudo da mãe $(\mathrm{p}=0,013)$, bem como com renda familiar $(\mathrm{p}<0,001)$. A mãe ter recebido informação anterior sobre prevenção de diarreia infantil mostrou associação estatística com idade materna $(\mathrm{p}<0,001)$. Conclusão: As mães apresentaram como principais habilidades para prevenir e manejar a diarreia infantil o encaminhamento da criança a um servicio de saúde, o uso de receitas caseiras e a inmunizaçao contra rotavirus, evidenciando que estas são situaçõnes nas quais os enfermeiros são capazes de intervir.
\end{abstract}

Palavras chave: Diarreia infantil, saúde da criança, comportamento materno, promoção da saúde, enfermagem.

\footnotetext{
ABSTRACT

Aim: To identify the maternal abilities in the prevention and handling of infantile diarrhea. Method: Transversal, quantitative study, carried out with 448 mothers of children under 5 years old, resident in Fortaleza-CE. Data

*Enfermeira. Enfermagem na Promoção da Saúde pela UFC Bolsista CNPq. Fortaleza, Brasil. Email: manujoventino@ yahoo.com.br

${ }_{* *}^{*}$ Enfermeira. Enfermagem na Promoção da Saúde pela UFC Professora da FAMETRO. Fortaleza, Brasil. Email: lydia_v_ freita@yahoo.com.br

${ }^{* * *}$ Enfermeira. Professora do Departamento de Enfermagem da UFC. Bolsista Produtividade do CNPq. Fortaleza, Brasil. Email: neivafrancenely@hotmail.com

${ }_{* * * *}$ Enfermeira. Enfermagem na promoção Bolsista PNPD. Fortaleza, Brasil. Email: priscilapetenf@yahoo.com.br

${ }^{* * * * *}$ Enfermeira. Professora do Departamento de Enfermagem da UFC. Fortaleza, Brasil. Email: anakarinaufc@hotmail.com

${ }^{* * * * *}$ Enfermeira. Professora do Departamento de Enfermagem da UFC. Bolsista CNPq Fortaleza, Brasil. Email: lbximenes@ yahoo.com.br
} 
were collected through interviews, using a form containing socio demographical characteristics and aspects related to maternal care for prevention and handling of infantile diarrhea. Results: The mothers had an average age of 28.34 years and 9.1 years of study. The medium income per capita was $\mathrm{R} \$ 150$. Most of the women had a couple and worked at home. Significant statistical correlation was observed between the realization of a homemade recipe and maternal age $(\mathrm{p}=0,027)$; and between the availability of oral rehydration solutions for the child and schooling of the mother $(\mathrm{p}=0,013)$. The previous act of receiving the information on prevention of infantile diarrhea showed statistical association with maternal age $(\mathrm{p}<0.001)$. Conclusion: The mothers has as main hability to prevent and handle infantile diarrhea the referal of the child to a health service, the use of homemade recipes and inmunization against rotavirus, showing that these are situations in which nurses are able to intervene.

Key words: Diarrhea infantile, child health, maternal behavior, health promotion, nursing.

\section{RESUMEN}

Objetivo: Identificar las habilidades maternas en la prevención y manejo de la diarrea infantil. Método: Estudio transversal, cuantitativo, realizado con 448 madres de niños menores de 5 años, en Fortaleza. Los datos fueron recolectados a través de entrevistas, utilizando un formulario que contenía datos sociodemográficos y aspectos relacionados a la atención materna para la prevención y manejo de la diarrea infantil. Resultados: Las madres tuvieron un promedio de 28,34 años y 9,1 años de estudio. La mediana de la renta per cápita fue de $\mathrm{R} \$ 150$. La mayoría tenía una pareja, trabajaba en casa y vivía hacía más de cinco años en la misma residencia. Hubo una correlación estadísticamente significativa entre la realización de medicinas caseras y la edad materna ( $\mathrm{p}$ $=0,027$ ); entre la oferta de solución de rehidratación oral para el niño y los años de estudio de la madre ( $\mathrm{p}=$ $0,013)$, así como con el ingreso familiar $(\mathrm{p}<0,001)$. La madre que había recibido información previa sobre la prevención de la diarrea infantil mostró asociación estadística con la edad ( $\mathrm{p}<0,001)$.La información previa recibida acerca de la prevención de la diarrea infantil mostró asociación estadística con la edad materna ( $\mathrm{p}$ $<0,001)$. Conclusión: Las madres tuvieron como principales habilidades para prevenir y manejar la diarrea infantil derivación del niño a un servicio de salud, el uso de recetas caseras y la inmunización contra el rotavirus, que demuestra que se trata de situaciones en las que las enfermeras pueden intervenir.

Palabras clave: Diarrea infantil, salud del niño, conducta materna, promoción de la salud, enfermería.

Fecha recepción: 15/03/12 Fecha aceptación: 22/03/13

\section{INTRODUÇÃO}

No decorrer das últimas décadas, o mundo presenciou mudanças consideráveis no que diz respeito ao conceito de saúde, passando de ausência de doenças para um completo estado de bem-estar biopsicosocial (1). Com este novo conceito, constata-se a inaptidão humana de manter permanente este estado de completo bem-estar, o que nos leva à necessidade de buscar constantemente o estado saudável.

Refletindo acerca deste panorama, a Carta de Ottawa traz dentro da Promoção da Saúde a responsabilidade dos profissionais de saúde de proporcionar meios para que a população possa melhorar o seu estado de saúde, e ainda capacitá-la para ser co-responsável pela manutenção destas condições (2).

Diante disto, constata-se que a população precisa ter habilidades para proporcionar e/ ou manter o seu próprio estado de saúde, de sua família e da comunidade. Contudo, tais habilidades podem, dependendo do momento, estarem presentes ou ausentes nos usuários do serviço de saúde, de forma que se faz necessário que o profissional de saúde, em especial o enfermeiro, verifique a existência dessas habilidades, visando o planeja- 
mento e execução de intervenções.

Para tanto, faz-se premente ressaltar que as estratégias para implementar a promoção da saúde são apoiadas por cinco domínios: construir políticas públicas saudáveis, criar ambientes favoráveis à sociedade, reforçar a ação comunitária para a saúde, reorientar os serviços de saúde e, por fim, desenvolver habilidades pessoais (3).

Destaca-se que as habilidades pessoais podem ter como alvo o autocuidado, o cuidado com a sua família ou o cuidado com as pessoas que estão próximas ao indivíduo. Contudo, em relação às crianças, estas dependem da habilidade de terceiros, em geral da mãe, para satisfazerem suas necessidades básicas, bem como para a prestação de cuidados diários.

Nesse contexto, a doença diarreica infantil se constitui em um dos principais agravos, no qual a habilidade materna em relação ao adequado manejo da doença de seus filhos influencia efetivamente na redução de suas complicações (4).

Inúmeros estudos demonstram intervenções comportamentais preventivas juntamente a mães e famílias de crianças que visam melhorar, por exemplo, habilidades quanto à lavagem das mãos (5) e relacionadas ao e tratamento da água destinada ao consumo humano $(6,7)$, reduzindo consideravelmente a ocorrência de episódios diarreicos entre crianças, bem como a duração destes.

Ressalta-se que a prevenção de doenças abrange medidas tanto para evitar a ocorrência da doença por meio da redução de fatores de risco (prevenção primária), quanto para evitar o seu progresso e reduzir as suas consequências (prevenção secundária e terciária), uma vez estabelecida (3).

Nesse contexto, ressalta-se que, na promoção da mudança comportamental ligada à saúde e desenvolvimento de habilidades, é preciso fornecer às pessoas, além de razões para mudar condutas de saúde, bem como meios e recursos para a efetivação de tais mudanças. Além disso, a pobreza e marginalização podem levar as pessoas a impossibilitar o exercício completo de escolhas autônomas; comprometendo a felicidade humana, bem como o processo de empowerment da comunidade (8).

Dessa forma, diante da oportunidade de conhecer as habilidades maternas para prevenir e conduzir a diarreia infantil, o enfermeiro poderá construir estratégias educativas no desenvolvimento destas habilidades de acordo com as necessidades identificadas. Nesta perspectiva, o presente estudo teve o objetivo relacionar as habilidades maternas na prevenção e manejo da diarreia infantil com as características sociodemográficas.

\section{MATERIALE E MÉTODO}

Trata-se de um estudo transversal, quantitativo, correlacional realizado com mães de crianças menores de 5 anos residentes em Fortaleza-CE. Fortaleza é dividida em seis Secretarias Executivas Regionais (SER), que são subsecretarias com suporte técnico, autonomia financeira e administrativa. Cada SER conta com uma estrutura de Centros de Saúde da Família (CSF), portas de entrada no sistema de saúde hierarquizado, contando com a assistência de equipes da Estratégia Saúde da Família para o acompanhamento da população adstrita.

No presente estudo foram selecionados seis CSF, pertencentes às SER III, IV e VI, escolhidas por se destacarem em relação ao número de crianças cadastradas com idade até 5 anos. De acordo com dados da Secretaria Municipal de Saúde, no ano de 2008, as SER III, IV e VI possuíam, respectivamente, $31.527,24.057$ e 40.386 crianças menores de 5 anos.

Assim, os critérios de inclusão no estudo foram: mães com pelo menos um filho(a) com idade inferior a 5 anos; e mães cujos filhos fossem acompanhados nos CSF sele- 
cionados pelo estudo. O critério de exclusão adotado foi a incapacidade cognitiva das mães que impossibilitasse a participação no estudo. Vale salientar que as mães que possuíam mais de um filho com idade inferior a 5 anos, convencionou-se como enfoque da investigação a criança com menor idade.

A amostragen foi do tipo não probabilística, por conveniencia e tamanho amostral foi baseado na fórmula para o cálculo de populações infinitas, totalizando 448 mães. A coleta de dados ocorreu de novembro de 2009 a fevereiro de 2010, por meio de entrevista, utilizando-se um formulário elaborado, validado e pré-testado pelos pesquisadores, abordando características sociodemográficas e aspectos relacionados ao cuidado materno para prevenção e manejo da diarreia em seus filhos, a saber: busca pelo serviço de saúde, realização e tipo de receita caseira, oferta anterior de soro de reidratação oral, imunização contra rotavírus e conduta materna quanto à alimentação da criança durante diarreia.

Ressalta-se que, em julho de 2009, o formulário para a entrevista passou pela validade aparente ou validade de rosto por meio de um pré-teste realizado com 30 mães de crianças menores de 5 anos que moravam na SER III. Tal etapa foi realizada após o consentimento livre e esclarecido das mães, por meio de visita domiciliária e da técnica da entrevista, visando à comodidade dos sujeitos. Após esta análise, alguns itens do formulário passaram por modificações, conforme as sugestões das mães participantes do pré-teste visando um melhor entendimento no momento da coleta de dados propriamente dita.

Durante a coleta de dados das 448 mães, as questões do formulário eram lidas e registradas por escrito pelos entrevistadores. Foran entrevistadas as mães que se, encontravan nas unidades de saúde de modo não aleatorio. As entrevistas ocorreram tanto pela manhã quanto pela tarde, em salas reservadas dos CSF, buscando-se garantir o conforto e a privacidade dos respondentes no momento da coleta de dados. Cada entrevista durou, em média, 14 minutos.

Vale resaltar que nem todas as mães responderam a totalidade do instrumento, já que muitas questões se relacionavan ao comportamento da mãe diante da diarreia de seu filho, e desta forma estas preguntas só poderiam ser respondidas pelas mães cujos filhos já tiviessem sido acometidos pela doença.

Os dados foram organizados e analisados através do Statistical Package for Social Science (SPSS), versão 17.0. Para a análise comparativa foi utilizado o teste Mann-Whitney, estabelecendo-se nível de significância inferior a 0,05. O estudo foi aprovado pelo Comitê de Ética em Pesquisa da Universidade Federal do Ceará, sob protocolo $n^{\circ}$ 92/09, sendo respeitadas todas as normas relacionadas às pesquisas envolvendo seres humanos.

\section{RESULTADOS}

A partir Tabela 1 pode-se observar que a maioria das mães entrevistadas tinha idade entre 19 e 29 anos ( $\mathrm{N}=240 ; 53,6 \%)$, com uma média de 28,34 anos (DP $\pm 7,67$ ), era casada ou vivia em união consensual $(\mathrm{N}=372$; $84,6 \%)$, trabalhava em casa, geralmente como donas de casa $(\mathrm{N}=307 ; 69 \%)$, possuíam de 7 a 10 anos de estudo $(\mathrm{N}=176 ; 39,6 \%)$, com média de 9,1 anos (DP $\pm 2,86$ ), e morava há mais de 5 anos na mesma residência $(\mathrm{N}=216 ; 48,2 \%)$. Além disso, na maioria dessas famílias a renda per capta mais prevalente foi até ${ }^{1} \mathrm{R} \$ 72$, mediana de $\mathrm{R} \$ 150,00$. Ressalta-se que a maioria das mães entrevistadas tinha apenas um filho vivo ( $\mathrm{N}=200 ; 45,2 \%)$, tendo predominado crianças do sexo feminino $(\mathrm{N}=225 ; 50,2 \%)$ (Tabela 1$)$.

Por meio da Tabela 2 pode-se verificar que prevaleceram as crianças que tinham apresentado algum episódio diarreico anterior $(\mathrm{N}=261 ; 58,3 \%)$, tendo sido levadas ao

${ }^{1} 1 \mathrm{USD}=\mathrm{R} \$ 2,29$ 
Tabela 1. Distribuição das mães participantes do estudo segundo características sociodemográficas. Fortaleza, 2011.

\begin{tabular}{|c|c|c|}
\hline Variáveis & $\mathbf{N}$ & $\%$ \\
\hline \multicolumn{3}{|l|}{ Idade $(\mathrm{N}=447)$} \\
\hline 14-19 & 42 & 9,4 \\
\hline $20-29$ & 231 & 51,6 \\
\hline $30-39$ & 137 & 30,6 \\
\hline $40-59$ & 37 & 8,3 \\
\hline \multicolumn{3}{|l|}{ Estado Civil $(\mathrm{N}=445)$} \\
\hline Casada/união consensual & 372 & 83,6 \\
\hline Outros & 73 & 16,4 \\
\hline \multicolumn{3}{|l|}{ Anos de Estudo $(\mathrm{N}=444)$} \\
\hline$\leq 6$ & 96 & 21,6 \\
\hline $7-10$ & 176 & 39,6 \\
\hline $11-13$ & 172 & 38,7 \\
\hline \multicolumn{3}{|l|}{ Trabalha fora de casa } \\
\hline Sim & 138 & 31 \\
\hline Não & 307 & 69 \\
\hline \multicolumn{3}{|c|}{ Renda per capita em salários mínimos $(\mathrm{N}=429)$} \\
\hline $0-1 / 4 \mathrm{SM}$ & 188 & 43,8 \\
\hline $1 / 4 \mathrm{SM}-1 / 2 \mathrm{SM}$ & 161 & 37,5 \\
\hline $1 / 2 S M-1 S M$ & 71 & 16,6 \\
\hline$\geq 1 \mathrm{SM}$ & 9 & 2,1 \\
\hline \multicolumn{3}{|c|}{ Pessoas residindo no domicílio $(\mathrm{N}=448)$} \\
\hline $2-3$ & 142 & 31,7 \\
\hline $4-5$ & 214 & 47,8 \\
\hline $6-11$ & 92 & 20,5 \\
\hline \multicolumn{3}{|c|}{ Tempo de moradia no mesmo lar em meses $(\mathrm{N}=443)$} \\
\hline$\leq 12$ & 94 & 21,2 \\
\hline $13-60$ & 138 & 31,2 \\
\hline$\geq 61$ & 211 & 47,6 \\
\hline \multicolumn{3}{|l|}{ Sexo do filho } \\
\hline Masculino & 223 & 49,8 \\
\hline Feminino & 225 & 50,2 \\
\hline \multicolumn{3}{|c|}{ Número de filhos vivos $(\mathrm{N}=442)$} \\
\hline 1 & 200 & 45,2 \\
\hline 2 & 127 & 28,7 \\
\hline $3-4$ & 97 & 21,9 \\
\hline$\underline{5-8}$ & 18 & 4,1 \\
\hline
\end{tabular}

Salário mínimo durante o estudo foi igual a R \$ 510,00.

Tabela 2. Distribuição das habilidades maternas no manejo da diarreia infantil e condições de saúde das crianças. Fortaleza, 2011.

\begin{tabular}{lrc}
\hline & $\mathrm{N}$ & $\%$ \\
\hline Existência anterior de algum episódio diarreico $(\mathbf{N}=\mathbf{4 4 7})$ & 261 & 58,3 \\
Sim & 186 & 41,7 \\
Não & & \\
Conduziu a criança com diarreia ao serviço de saúde $(\mathbf{N}=\mathbf{2 6 0})$ & 208 & 80 \\
Sim & 52 & 20 \\
Não & & \\
Realização de receita caseira quando a criança encontra-se com diarreia $(\mathbf{N}=\mathbf{2 5 9})$ & 150 & 57,9 \\
Sim & 109 & 42,1 \\
Não
\end{tabular}


Continuação Tabela 2.

\begin{tabular}{lrc}
\hline Receita caseira utilizada (N=150) & & \\
soro caseiro & 120 & 80 \\
chá do olho da goiabeira & 8 & 5,3 \\
fruta constipante & 7 & 4,7 \\
Outros & 15 & 10 \\
Oferta anterior de SRO para o filho (N=261) & 173 & 66,3 \\
Sim & 88 & 33,7 \\
Não & & \\
Conduta materna quanto à alimentação da criança durante diarreia $(\mathbf{N}=\mathbf{2 5 1})$ & 73 & 29,1 \\
Suspende a alimentação normal da criança & 61 & 24,3 \\
Continua oferecendo a mesma alimentação & 117 & 46,6 \\
Procura melhorar a alimentação e hidratar mais & & \\
Imunização contra rotavírus (N=448) & 311 & 69,4 \\
Sim & 103 & 23 \\
Não & 34 & 7,6 \\
Outros & & \\
\hline
\end{tabular}

serviço de saúde ( $\mathrm{N}=208 ; 52 \%)$. Contudo, dessas 261 crianças que haviam tido diarreia anterior, 150 (57,9\%) receberam algum tipo de receita caseira, sendo em sua maioria o soro caseiro $(\mathrm{N}=120 ; 80 \%)$.

Entre as receitas caseiras citadas pelas mães, identificou-se o uso de alguns alimentos ditos considerados constipantes pelas mesmas, como: banana, goiaba, maçã, beterraba e cenoura. Além de oferecerem água de coco, chá de boldo, chá de casca de laranja, gergelim com mostarda, chá de hortelã com canela, entre outros.

Observou-se que apenas 311 (75,1\%) crianças haviam recebido a vacina contra o rotavírus.

A maioria das mães $(\mathrm{N}=117 ; 46,6 \%)$ referiu que procura melhorar a alimentação da criança e hidratá-la mais na ocasião dos episódios diarreicos em seus filhos.

Verificou-se que não houve associação estatisticamente significante entre o estado civil da mãe e suas habilidades de cuidado com o filho, visando à prevenção e ao manejo da diarreia. Da mesma forma, não se encontrou significância estatística entre o fato de a mãe trabalhar fora de casa e suas habilidades de cuidado relacionadas à diarreia infantil.

Contudo, verificou-se correlação estatisticamente significante entre a reali- zação de receita caseira e idade materna $(p=0,027)$; entre oferta de solução de reidratação oral (SRO) para a criança e anos de estudo da mãe $(p=0,013)$, bem como com renda familiar $(\mathrm{p}<0,001)$. A mãe ter recebido informação anterior sobre prevenção de diarreia infantil mostrou associação estatística com idade materna $(\mathrm{p}<0,001)$.

\section{DISCUSSÃO E CONCLUSÃO}

Pesquisas têm verificado que o reduzido conhecimento materno, sua baixa escolaridade e a pouca idade são aspectos que influenciam no adoecimento de crianças por doenças diarreicas agudas, podendo ser associados à dificuldade para entender atividades educativas, limitando a busca dos benefícios necessários em função da pouca ou nenhuma experiência, levando também à ineficácia do cuidar, sobretudo em relação à higiene e à alimentação. Indiretamente, o baixo nível de escolaridade também influencia o desemprego das mães/cuidadoras, elevando, assim, a desigualdade sócio-econômica $(9,10)$.

Além disso, a idade materna pode estar associada ao recebimento de orientações anteriores acerca das medidas preventivas e 
do manejo da diarreia infantil, fato esperado tendo em vista que quanto mais tempo de vida um indivíduo tiver, maior probabilidade de ser exposto a certos eventos.

Estudo conduzido no Norte e Nordeste do Brasil verificou que crianças cujas mães tinham escolaridade igual ou inferior a 3 anos de estudo, residentes em domicílios com renda familiar igual ou inferior a 1 salário mínimo e com mais de 5 pessoas apresentaram frequência maior de geo-helmintíases, parasitos fortemente associados com a ocorrência de diarreia (11).

A maioria das mães era casada ou vivia em união consensual e não possuía ocupação fora do lar, contudo tais fatores não mostraram associação com suas habilidades para cuidar de seus filhos na prevenção e tratamento de diarreia. Assim, infere-se que o cuidado que a mãe presta ao seu filho não está relacionado à existência de um companheiro, bem como que mesmo inserida no mercado de trabalho, a mãe pode oferecer cuidado de qualidade para os seus filhos no que diz respeito à diarreia.

Verificou-se que a maioria das famílias vivia abaixo da linha da pobreza, pois a renda per capita de zero a $1 / 4$ do salário mínimo $(\mathrm{R} \$$ 127,50) predominou. Estudos demonstram que a renda familiar mensal igual ou inferior a 1 salário mínimo influencia a ocorrência de helmintíases (11), bem como da própria diarreia infantil (10). Corroborando com este fato, estudo de caso-controle realizado na Etiópia também demonstrou que quanto mais elevada a renda familiar maior fator protetor essa variável confere em relação à prevenção de diarreia infantil (12).

Estudo realizado, de 2007 a 2008, na Etiópia incluindo 1516 pessoas acerca do uso de intervenções para a prevenção da diarreia infantil encontrou uma média de 4,8 moradores por domicílio, assemelhando-se à presente pesquisa, cujo achado foi de 4,45. Sabe-se que quanto maior o número de moradores em um domicílio, maior o risco para a aquisição de doenças, especialmente, infec- to-contagiosas como a diarreia (6). Possivelmente este fato esteja associado a residências com poucos cômodos, dificultando, assim, as práticas preventivas de diarreia infantil, como higiene pessoal e limpeza doméstica.

Quanto ao tempo de moradia em um mesmo lar verificou-se uma média de 9,25 anos, podendo ser considerado um fator positivo já que Teixeira e Heller (13) referem que essa variável associa-se com a ocorrência de diarreia, de forma que quanto maior o tempo de residência da família na moradia, maior o investimento dos moradores na melhoria de suas condições de habitabilidade, inclusive em relação às instalações sanitárias domiciliares.

Em relação ao número de filhos, acreditase que quanto maior o número de filhos, mais dedicação os cuidadores terão que demandar para prevenir eficazmente a diarreia infantil, podendo, no presente estudo, ser um fator que favorece este cuidado materno tendo em vista que a maioria das crianças eram filhos únicos.

A maioria das crianças estudadas já tinha apresentado algum episódio diarreico, em relação a isso, adéqua-se o conceito de autoeficácia por relacionar-se às crenças que as pessoas têm para transformar suas ações, influenciando nos eventos que afetam suas vidas, na quantidade de esforço que será dispensada e de tempo em que irão persistir para transpor obstáculos e experiências adversas (14). Nesse sentido, acredita-se que uma experiência anterior pode influenciar na autoeficácia materna para manejar adequadamente outros episódios posteriores de diarreia em seus filhos.

Possivelmente, o número de casos notificados de diarreia seja superior ao divulgado oficialmente, pois há uma deficiência no registro de dados, uma vez que não se trata de uma condição de doença, cuja notificação é compulsória. Além disso, o fato de as mães não levarem os filhos ao serviço de saúde, bem como o tratamento caseiro contribuem para a subnotificação, mascarando as esta- 
tísticas das ocorrências de adoecimento por diarreia (10).

Muitas vezes as mães realizam receitas caseiras para tratar a diarreia, estando associado no estudo com a idade materna. Dessa forma, acredita-se que quanto mais idade esta possua, maior será sua autoeficácia na prevenção e no manejo da diarreia infantil. Contudo, há que se ter atenção em relação a este tipo de cuidado, pois algumas receitas caseiras não possuem eficácia cientificamente comprovada, constituindo-se em um risco para a saúde da criança.

Metanálise conduzida com 157 artigos verificou que a SRO é capaz de prevenir 93\% das mortes por diarreia (15). Por conta dessa eficácia, os pais devem estar cientes dos riscos que a gastroenterite acarreta aos seus filhos, bem como ser familiarizado com o uso de SRO. Estas soluções devem ser mantidas acessíveis a todas as casas que têm crianças, sendo o papel do pessoal de enfermagem ensinar sobre como lidar com a diarreia aguda, tanto em relação à prevenção quanto ao tratamento precoce (16).

$\mathrm{O}$ presente estudo encontrou associação estatísticamente significante entre a oferta de SRO e a escolaridade materna e que quanto maior a renda maior a probabilidad de a mãe ofrecer o SRO para seu filho, podendo estar associado ao fato de que torna-se mais simples para essas mulheres empoderar-se das orientações prestadas pelos profissionais de saúde acerca da função e do preparo do SRO.

Estudo realizado na República Checa verificou um baixo conhecimento materno sobre a SRO (27,6\%), além do baixo uso do mesmo ( $1,9 \%$ durante o último episódio diarreico do filho) (17). Em Bangladesh, das 377 mães que demonstraram a preparação de SRO, $237(56,4 \%)$ o fizeram de maneira incorreta, sendo este fato associado significativamente com a recusa de SRO por parte das crianças. No referido estudo, preparo anterior do SRO esteve associado à uma diluição correta (18).

Sabe-se que inúmeras internações infantis são devido à diarreia viral, sendo o principal causador o rotavírus, o qual é responsável, a cada ano, por 114 milhões de episódios de diarreia; 2,4 milhões de admissões hospitalares e 600 mil mortes em crianças menores de 5 anos em todo o mundo (19).

No presente estudo pode-se verificar que 311 (69,4\% mães levaram seus filhos para serem vacinados contra esse virus. Sabe-se que no Brasil, a vacina contra o rotavírus foi introduzida em 2006, tendo sido comprovada a eficácia da mesma na redução dos custos com internações e consultas ambulatoriais no nordeste brasileiro (20). Além disso, o pacote de prevenção de diarreia da UNICEF e OMS (21) destaca a necessidade de incentivo à vacinação contra o rotavírus, devido à sua comprovada eficácia $(22,23)$. Assim, é essencial que a mãe ou familiar leve a criança ao centro de saúde mais próximo e que seja respeitado o aprazamento correto da vacina, visando à prevenção da doença, haja vista a vulnerabilidade da criança às infecções que potencializam a ocorrência e a gravidade dos episódios diarreicos (24).

Quanto à conduta materna em relação à alimentação da criança durante o episódio diarreico, verificou-se que a maioria das mães referiu realizar a melhoria no padrão de alimentação e de hidratação da criança, sendo que o Ministério da Saúde recomenda que não seja modificada a dieta e que se aumente ou introduza a oferta de líquidos durante a diarreia (25).

Estudo realizado na rede pública do município de São Paulo corroborou com estes achados, tendo em vista que a maioria dos entrevistados tinha consciência sobre a importância do aumento da oferta de líquidos durante os episódios diarreicos, entretanto, com relação à alimentação, $100 \%$ dos respondentes associaram a necessidade de pausa ou dieta alimentar como cuidado que deveria ser prestado à criança com diarreia, embora essa conduta seja totalmente superada, do ponto de vista científico (26). Kudlova (17) encontrou em seu estudo que a manuten- 
ção da alimentação rotineira foi rara $(5,2 \%)$, tendo sido a dieta da criança modificada em $75,2 \%$ durante o período diarreico.

Todos estes aspectos constituem aptidões para a vida, que consistem em habilidades de comportamento adaptativo e positivo, que permitem aos indivíduos lidarem eficazmente com as demandas e os desafios da vida cotidiana. Qualquer atividade realizada por um indivíduo, independentemente do seu estado de saúde real ou percebido, com a finalidade de promover, proteger ou manter a saúde; tal comportamento é objetivamente eficaz para esse fim (14).

Assim, o desenvolvimento de habilidades pessoais dessas mães é essencial para que se alcancem melhorias na promoção da saúde infantil, devendo estas ser visadas pelo enfermeiro. Estas habilidades podem ser obtidas mediante a divulgação de informação, educação para a saúde e intensificação das habilidades vitais, para que as populações possam exercer maior controle sobre sua própria saúde (2).

Em conclusão habilidades maternas para a prevenção da diarreia infantil constituemse em aspectos relevantes para o processo de trabalho do enfermeiro, o qual somente poderá intervir adequadamente se tiver conhecimento destas.

Nesse sentido, a maioria das mães investigadas tinha de 19 a 29 anos, era casada ou vivia em união consensual, trabalhava no próprio lar, possuía de 7 a 10 anos de estudo, morava há mais de 5 anos na mesma residência e possuía renda per capta de até $\mathrm{R} \$ 72$. Quanto às suas habilidades no contexto do manejo da diarreia de seus filhos, verificou-se que prevaleceram aquelas que levaram-nos ao serviço de saúde durante os episódios da doença, ofereceram algum tipo de receita caseira, como o soro caseiro e outros alimentos ditos constipantes pelas mesmas.

Verificou-se correlação estatisticamente significante entre a realização de receita caseira e idade materna $(p=0,027)$; entre oferta de SRO para a criança e anos de estudo da mãe $(p=0,013)$, bem como com renda familiar $(p<0,001)$. A mãe ter recebido informação anterior sobre prevenção de diarreia infantil mostrou associação com idade materna $(\mathrm{p}<0,001)$.

Diante das habilidades maternas na prevenção da diarreia infantil, cabe ao enfermeiro desenvolver estratégias que visem orientar às mães de crianças com idade inferior a cinco anos sobre como realizar o correto manejo desta doença, visando a promoção da saúde desta população.

\section{REFERÊNCIAS}

1. World Health Organization. Constitution of the World Health Organization, Basic Documents. WHO: Genebra; 1946.

2. World Health Organization. Ottawa Charter for Health Promotion: First International Conference on Health Promotion. Ottawa, Canada: WHO; 1986. (WHO/HPR/HEP/95.1).

3. Nutbeam D. Health Promotion Glossary. Health Promot. Int. 1998; 13(4): 349-64.

4. Vanderlei LCM, Silva GAP. Diarreia aguda: o conhecimento materno sobre a doença reduz o número de hospitalizações nos menores de dois anos? Rev Assoc Med Bras. 2004; 50(3): 276-81.

5. Ejemot RI, Ehiri JE, Meremikwu MM, Critchley JA. Hand washing for preventing diarrhoea. Cochrane Database Syst Rev [Internet]. 2008 [citada 30 nov 2010]; (1). Disponível em: http://onlinelibrary.wiley.com

6. Boisson S, Schmidt WP, Berhanu T, Gezahegn H, Clasen T. Randomized Controlled Trial in Rural Ethiopia to Assess a Portable Water Treatment Device. Environ Sci Technol. 2009; 43(15): 5934-9.

7. Tiwari SSK, Schmidt WP, Darby J, Kariuki $Z G$, Jenkins $M W$. Intermittent slow sand filtration for preventing diarrhea 
among children in Kenyan households using unimproved water sources: randomized controlled trial. Trop Med Int Health. 2009; 14(11): 1374-82.

8. Shuftan C. An ethical question: Are health professionals promotors of the status quo or of social change? Promot Educ. 2008; 15(3): 30-3.

9. Gomes DKM, Lucena MC, Barros MG. Perfil epidemiológico e coproparasitológico de crianças menores de 5 anos internadas no hospital governador João Alves Filho em Aracajú - SE, com quadro de diarreia aguda. Ver. Bras. Anal. clin. 2005; 37(4): 257-9.

10. Pereira IV, Cabral IE. Diarreia aguda em crianças menores de um ano: subsídios para o delineamento do cuidar. Esc. Anna Nery Rev. Enferm. 2008; 12(2): 224-9.

11. Fonseca EOL, Teixeira MG, Barreto ML, Carmo EH, Costa MCN. Prevalência e fatores associados às geo-helmintíases em crianças residentes em municípios com baixo IDH no Norte e Nordeste brasileiros. Cad Saude Publica. 2010; 26(1): 14352.

12. Mediratta RP, Feleke A, Moulton LH, Yifru S, Sack RB. Risk Factors and Case Management of Acute Diarrhoea in North Gondar Zone, Ethiopia. J Health Popul Nutr. 2010; 28(3): 253-63.

13. Teixeira JC, Heller L. Fatores ambientais associados à diarreia infantil em áreas de assentamento subnormal em Juiz de Fora, Minas Gerais. Rev Bras Saude Mater. Infant. 2005; 5(4): 449-55.

14. Smith BJ, Tang KC, Nutbeam D. WHO health promotion glossary: new terms. Health Promot. Int. 2006; 21(4): 340-5.

15. Munos MK, Walker CL, Black RE. The effect of oral rehydration solution and recommended home fluids on diarrhea mortality. Int J Epidemiol. 2010; 39(Suppl 1): i75-87.
16. Casado MJ. Acute infantile diarrhea. Nursing treatment. Rev Enferm. 2010; 33(9): 50-9.

17. Kudlova E. Home management of acute diarrhoea in Czech children. J Pediatr Gastroenterol Nutr. 2010; 50(5): 510-5.

18. Ahmed FU, Rahman ME, Mahmood CB. Mothers' skills in preparing oral rehydration salt solution. Indian J Pediatr. 2000; 67(2): 99-102.

19. Grimwood K, Buttery JP. Clinical update: rotavírus gastroenteritis and its prevention. Lancet. 2007; 370: 302-4.

20. Centenaria C, Gurgelb RQ, Bohland AK, Oliveira DMP, Faragherd B, Cuevas LE. Rotavirus vaccination in northeast Brazil: A laudable intervention, but can it lead to cost-savings? Vaccine. 2010; 28(25): 4162-8.

21. The United Nations Children's Fund (UNICEF), World Health Organization (WHO). Diarrhoea: Why children are still dying and what can be done. Geneve: WHO; 2009. 60 p.

22. Parashar UD, Glass RI. Rotavirus vaccines early success, remaining questions. $\mathrm{N}$ Engl J Med. 2009; 360(11): 1063-5.

23. Pont SJ, Carpenter LR, Griffin MR, Jones TF, Schaffner W, Dudley JA et al. Trends in healthcare usage attributable to diarrhea, 1995-2004. J Pediatr. 2008; 153(6): 777-82.

24. Diggle L. Rotavirus diarrhoea and future prospects for prevention. Br J Nurs. 2007; 16(16): 970-4.

25. Ministério da Saúde Brasil. AIDPI Atenção Integrada às Doenças Prevalentes na Infância: curso de capacitação: introdução: módulo 1. $2^{\mathrm{da}}$ ed. Brasilia: Ministério da Saúde; 2002.

26. Prado SRLA, Fujimori E. Conhecimento materno/familiar sobre o cuidado prestado à criança doente. Rev Bras Enferm. 2006; 59(4): 492-6. 